\title{
PEDESTRIAN LEVEL OF SERVICE CRITERIA FOR URBAN OFF-STREET FACILITIES IN MID-SIZED CITIES
}

\author{
Rima Sahani, Prasanta Kumar Bhuyan \\ Dept of Civil Engineering, National Institute of Technology Rourkela, India \\ Submitted 14 February 2014; accepted 2 May 2014; \\ first published online 10 September 2014
}

\begin{abstract}
Levels Of Service (LOS) evaluation criteria for off-street pedestrian facilities are not well defined in urban Indian context; hence an in-depth research is carried out in this regard. Defining Pedestrian Level of Service (PLOS) criteria is basically a classification problem; therefore a comparative study is made using three methods of clustering i.e. Affinity Propagation (AP), Self-Organizing Map (SOM) in Artificial Neural Network (ANN) and Genetic AlgorithmFuzzy (GA-Fuzzy) clustering. Pedestrian data are used on validation measure of clustering method to obtain optimal number of cluster used in defining PLOS categories. To decide the most suitable algorithm applicable in defining PLOS criteria for urban off-street facilities in Indian context, Wilk's Lambda is used on results of the three clustering methods. It is observed from the analysis that GA-Fuzzy is the most suitable clustering analysis among the three methods. With the help of GA-Fuzzy clustering analysis the ranges of the four measuring parameters (average pedestrian space, flow rate, speed of pedestrian and volume to capacity ratio) are defined by using the data collected from two mid-sized cities located in the state of Odisha, India. It is also observed that at $>16.53 \mathrm{~m}^{2} /$ ped average space, $\leq 0.061 \mathrm{ped} / \mathrm{sec} / \mathrm{m}$ flow rate, $>1.21$ speed and $\leq 0.34 \mathrm{v} / \mathrm{c}$ ratio pedestrians can move in their desired path at LOS ' $\mathrm{A}$ ' without changing movements and it is the best condition for off-street facilities. But in the pedestrian facility having $\leq 4.48 \mathrm{~m}^{2} /$ ped average space, $>0.146 \mathrm{ped} / \mathrm{sec} / \mathrm{m}$ flow rate, $\leq 0.62$ average speed and $>1.00 \mathrm{v} / \mathrm{c}$ ratio, pedestrian movement is severely restricted and frequent collision among users occurs. The ranges of the parameters used for LOS categories found in this study for Indian cities are different from that mentioned in HCM (Highway Capacity Manual 2010) because of differences in population density, traffic flow condition, geometric structure and some other factors.
\end{abstract}

Keywords: off-street pedestrian facility; pedestrian level of service; affinity propagation; self-organizing map; genetic algorithm-fuzzy; clustering validation.

\section{Introduction}

Levels Of Service (LOS) is a complex term which represents the operating condition of pedestrian facilities and level of comfort pedestrian experience in using these facilities as LOS indirectly represents the actual feeling of the people. With the exponential increase in motorization, limited attention has been paid to pedestrian and public transport facilities. A change in focus is required which will allow people, not vehicles, to reclaim the urban environment. Growing motorization has also lead to a dramatic increase in the number of pedestrian fatalities and accidents, and high levels of air pollutionparticularly exposing pedestrians who walk to work or access public transport to reach their destinations. There are few initiatives to promote the improvement of walking in mid-sized cities. The few civil society organizations and non-government organizations working in this area can play key roles in promoting improvements on walkability and pedestrian facilities in their cities.
As Pedestrian Level of Service (PLOS) is not well defined for highly heterogeneous traffic flow on urban corridors in countries like India, an attempt has been made to define LOS criteria in this study. Defining the PLOS criteria is a module of LOS analysis procedure of urban off-street pedestrian facilities. These methodologies affect the planning, design, and operational aspects of transportation projects as well as the allocation of limited financial resources among competing transportation projects. This envisages the importance of suitable methods that should be adopted while defining the PLOS criteria of urban streets in the context of cities in India. Considering the importance of LOS analysis for urban off-streets pedestrian facilities in Indian context, an in-depth research was carried out in the present study.

In this study two important mid-sized cities (population less than a million), Bhubaneswar and Rourkela of Odisha state (India) are taken as the study area. By using video data collection procedure all the field data

Corresponding author: Prasanta Kumar Bhuyan

E-mails: bhuyanp@nitrkl.ac.in; pkbtrans@gmail.com 
like speed of pedestrian, effective walkway width and pedestrian hourly volume are collected. For determination of different LOS categories parameters like flow rate, pedestrian space and $v / c$ ratio are calculated. Then with the help of three clustering methods i.e. Self-Organized Map (SOM) clustering in Artificial Neural Network (ANN), Affinity propagation (AP) clustering and Genetic Algorithm (GA) Fuzzy clustering, six different ranges of PLOS are determined.

\section{Background of the Study}

HCM (Highway Capacity Manual 2000) describes that the LOS criteria for pedestrian flow are based on subjective measure, which can be imprecise. However, it is possible to define ranges of space per pedestrian, flow rates and speeds, which then can be used to develop quality-of-flow criteria. It defines six LOS categories for pedestrian facilities. The HCM (Highway Capacity Manual 2010) also designates six LOS from 'A' to ' $F$ ', for pedestrian facility, with LOS 'A' representing the best operating conditions and LOS ' $\mathrm{F}$ ' the worst. It uses distinct average pedestrian space values as boundaries for the various LOS. HCM (Highway Capacity Manual 2010) stated that as volume and density increase, pedestrian speed declines. As density increases and pedestrian space decreases, the degree of mobility afforded to the individual pedestrian declines, as does the average speed of the pedestrian stream. According to HCM (Highway Capacity Manual 2000), pedestrian facilities can be of two types: uninterrupted and interrupted. When pedestrian facilities are not affected by any motorized modes of travel then the facility is known as the uninterrupted pedestrian facility or off-street pedestrian facility and vice versa. Several studies have been performed relating to the analysis of PLOS of pedestrian facilities. Jaskiewicz (2000) stated that in order to encourage walking as a viable alternate form of transportation, it is essential that careful attention be paid to pedestrian comfort and safety in addition to traditional volume and capacity factors. Muraleetharan and Hagiwara (2007) developed a methodology to estimate the overall LOS of pedestrian walkways and crosswalks on the concept of total utility value. The authors suggested that the efforts to accommodate pedestrians in urban areas should focus on improving the walking environment of the road network.

Petritsch et al. (2006) incorporated traffic volumes on the adjacent roadway and exposure (i.e. crossing widths) at conflict points with intersections and driveway and the study reveals that traffic volumes on the adjacent roadway and the density of conflict points along the facility are the primary factors in the LOS model for pedestrians traveling along urban arterials with sidewalks. Laxman et al. (2010) have carried out a study for medium-sized cities in India and analysed the pedestrians flow characteristics under mixed traffic condition. Sisiopiku et al. (2007) compared the various pedestrian sidewalk assessments and shown that the same sidewalk segment may receive multiple LOS ratings when different assessment methods are considered and the fact applies to sidewalks located on both urban and campus-like environments. Van Houten et al. (2007) stated that because pedestrian signal violations at midblock crosswalks are associated with pedestrian crashes, it is important to improve pedestrian signal compliance at these locations. One way to improve compliance is to decrease pedestrian delay by reducing minimum green time. Miller et al. (2008) investigated the approach speed and passing clearance that Seg-way devices exhibit on encountering a variety of obstacles on the sidewalk. Kim et al. (2006) presented a sidewalk pavement width design method for making more pedestrian friendly and walk-inspiring sidewalk pavements in the urban area investigating the pedestrians' preferences on the levels of service, surveyed actual foot path trajectories in the sidewalk pavements, and observed pedestrian movement characteristics in the streets. Rastogi et al. (2011) have shown different average walking speeds observed in different countries like in United States $-81 \mathrm{~m} / \mathrm{min}$, France $-90 \mathrm{~m} / \mathrm{min}$, Israel - $79 \mathrm{~m} / \mathrm{min}$, Canada $-84 \mathrm{~m} /$ min, Sri Lanka $-75 \mathrm{~m} / \mathrm{min}$, China $-74 \mathrm{~m} / \mathrm{min}$ and India $-72 \mathrm{~m} / \mathrm{min}$. As a point of comparison using two measuring parameters such as average pedestrian space $\left[\mathrm{m}^{2} / \mathrm{ped}\right]$ and flow rate $[\mathrm{ped} / \mathrm{m} / \mathrm{min}]$, Table 1 shows PLOS for different countries as prepared by US Federal Highway Administration (Rouphail et al. 1998).

Table 1. Walkways LOS thresholds of different countries (Rouphail et al. 1998)

\begin{tabular}{|c|c|c|c|c|}
\hline \multirow{2}{*}{$\begin{array}{c}\text { LOS } \\
\text { categories }\end{array}$} & \multicolumn{4}{|c|}{ Pedestrian space $\left[\mathrm{m}^{2} / \mathrm{ped}\right]$} \\
\hline & USA (HCM) & Germany & Israel & Thailand \\
\hline 'A' & 12 & 10 & - & 2.38 \\
\hline 'B' & $3.7 \div 12$ & $3.3 \div 10$ & - & $1.60 \div 2.38$ \\
\hline 'C’ & $2.2 \div 3.7$ & $2 \div 3.3$ & 1.67 & $0.98 \div 1.60$ \\
\hline ‘D’ & $1.4 \div 2.2$ & $1.4 \div 2$ & $\begin{array}{c}1.33 \div 1.66 \\
0.8 \div 1.33\end{array}$ & $0.65 \div 0.98$ \\
\hline 'E’ & $0.6 \div 1.4$ & $0.6 \div 1.4$ & $0.5 \div 0.8$ & $0.37 \div 0.65$ \\
\hline \multirow[t]{2}{*}{ 'F' } & 0.6 & 0.6 & - & 0.37 \\
\hline & \multicolumn{4}{|c|}{ Pedestrian flow rate $[\mathrm{ped} / \mathrm{m} / \mathrm{min}]$} \\
\hline 'A' & 6.6 & - & - & 28 \\
\hline ‘B’ & $6.6 \div 23$ & - & & $28 \div 40$ \\
\hline 'C' & $23 \div 33$ & - & 40 & $40 \div 61$ \\
\hline ‘D’ & $33 \div 49$ & - & $\begin{array}{l}40 \div 50 \\
50 \div 75\end{array}$ & $61 \div 81$ \\
\hline 'E’ & $49 \div 82$ & - & $75 \div 95$ & $81 \div 101$ \\
\hline 'F' & var. & - & - & 101/var. \\
\hline
\end{tabular}

Cluster analysis or clustering is the task of grouping a set of objects in such a way that objects in the same group (called cluster) are more similar (in some sense or another) to each other than to those in other groups (clusters). Objects in a cluster are closer to the 'centre' of a cluster, than to the centre of any other cluster. Čokorilo et al. (2014) addressed the problem of aircraft accident using general screening with the help of cluster analysis. The authors revealed that cluster analysis can be used to discover structures in data without providing an ex- 
planation/interpretation. From the two types of data partitions exist in cluster analysis: (1) strong or precise (crisp); (2) weak or blurred (fuzzy), the authors used the crisp partition in the study. A good clustering method will produce clusters with the property that their intracluster distance is small and their inter-cluster distance is large (Kaufman, Rousseeuw 2005). AP is a theoretic clustering method recently developed by Frey and Dueck (2007). This algorithm simultaneously considers all of data points as possible exemplar (centre point) where each message is sent to reflect the latest interest which is owned by each data point to be able to select another data points as their exemplar. In recent past researchers have used this efficient and accurate algorithm in solving various clustering problems. Dueck and Frey (2007) used AP algorithm to cluster images of faces and genes in microarray data. The authors found AP to cluster data with much lower error than $k$-centres cluster, and it did the clustering in less than one-hundredth the amount of time. Xia et al. (2008) present two variants of AP for grouping large scale data with a dense similarity matrix. The local approach was Partition Affinity Propagation (PAP) and the global method was Landmark Affinity Propagation (LAP).

Many researchers have utilized GA in optimizing the clustering problems. Zhou and Khotanzad (2007) developed a Genetic Fuzzy Clustering Algorithm combining Fuzzy C-Mean (FCM) clustering and GA. Fuzzy clustering is a process in which a data point is not assigned to a single cluster, rather each data points possess a membership function. This membership function indicates the strength of the data point. It can be noted that fuzzy clustering has two main advantages over other methods in its application to solve such kind of classification problems. Firstly, memberships can be combined with other information. In particular, in the special case where memberships are probabilities, results can be combined from different sources using Bayes' theorem. Secondly, the memberships for any given object indicate whether there is a second best cluster that is almost as good as the best cluster, a phenomenon which is often hidden when using other clustering techniques.

GA is used in traffic engineering field by various researchers to solve traffic engineering problems. Lingras (2001) utilized GA to estimate the missing traffic count. $50 \%$ of permanent traffic counts have missing data. The researchers designed a genetically designed regression model having very high precision. Fang and Pecheux (2009) studied about the LOS of a signalized intersection taking user perception into account. Unsupervised data clustering technique such as fuzzy $c$-means clustering was used to get distinct cluster of user perceived delay and service rating. Clustering result was analyzed according to approach membership, delay membership and rating membership. SOM is one of the ANN having the inherent capability to learn the pattern of input and to detect regularities and correlations in their input and responses in future accordingly. The application of this particular problem to define the LOS of urban street ANN is used for clustering of speed data. Lingras (1995) compared grouping of traffic pattern using the Hierar- chical Agglomerative Clustering and the Kohonen Neural Network methods in classifying traffic patterns. It is advantageous to use hierarchical grouping on a small subset of typical traffic patterns to determine the appropriate number of groups and change its parameters to reflect the changing traffic patterns. Such an approach is useful in using hour-to-hour and day-to-day traffic variations in addition to the monthly traffic-volume variation in classifying highway sections.

From the above background study it has been seen that, there is a significant body of research featuring new ways of evaluating pedestrian service levels on urban sidewalks. These studies recommend everything from small amendments to the HCM's LOS calculation to completely new LOS methodologies, depending on local needs and characteristics. These studies suggest that the current tool for measuring pedestrian LOS prescribed by the HCM may not take into account important differences in pedestrian characteristics, location characteristics, and flow characteristics when evaluating Indian City sidewalks. If that is the case, the LOS used to evaluate Indian cities sidewalks does not serve the city's pedestrians. The issue is how to determine the threshold values for partitioning different PLOS categories. AP, GA Fuzzy and SOM clustering are some of the clustering methods that are applied to define urban off-street pedestrian LOS categories in this study. The major objectives of this study are: (1) to classify urban off streets pedestrian LOS categories and (2) to select the best cluster analysis method applicable in defining PLOS criteria for mid-sized urban off-streets facility in Indian context.

\section{Cluster Analysis Methods and Validation Measures}

AP, GA Fuzzy and SOM in ANN clustering applied to define PLOS are discussed below.

\subsection{Affinity Propagation (AP) Clustering}

AP is a theoretic clustering method recently developed by Dueck and Frey (2007). This algorithm simultaneously considers all of data points as possible exemplar (centre point) where each message is sent to reflect the latest interest which is owned by each data point to be able to select another data points as their exemplar. Procedurally, the algorithm operates on three matrices: a similarity $(s)$ matrix, a responsibility $(r)$ matrix, and availability $(a)$ matrix. Results are contained in a criterion $(c)$ matrix. These matrices are iteratively updated by four equations, where $i$ and $k$ refer, respectively, to the rows and columns of the associated matrix.

Steps:

1. Input similarity matrix $s(i, k)$ : the similarity of point $i$ to point $k$;

2. Initialize the availabilities $a(i, k)$ to zero: $a(i, k)=0$;

3. Updating all responsibilities $r(i, k)$ :

$$
r(i, k) \leftarrow s(i, k)-\max \left\{a\left(i, k^{\prime}\right)+s\left(i, k^{\prime}\right)\right\} ;
$$


4. Updating all availabilities $a(i, k)$ :

$a(i, k) \leftarrow \min \left\{0, r(k, k)+\sum_{i^{\prime}: i^{\prime} \notin\{i, k\}} \max \left\{0, r\left(i^{\prime}, k\right)\right\}\right\}$, for $k \neq i$;

5. Availabilities and responsibilities matrix were added to monitor the exemplar decisions. For a particular data point $i ; a(i, k)+a(i, k)>0$ for identification exemplars;

6. If decisions made in step 3 did not change for a certain times of iteration or a fixed number of iteration reaches, go to step 5. Otherwise, go to step 1;

7. Assign other data points to the exemplars using the nearest assign rule that is to assign each data point to an exemplar which it is most similar to.

\subsection{GA-Fuzzy Clustering}

As followed by the article written by Zhou and Khotanzad (2007) GA-Fuzzy algorithm is a combination of FCM and GA. For clustering of data FCM algorithm is used. The ability GA in finding global minimum is utilized in this hybrid algorithm to get an optimal clustering result.

\subsubsection{FCM Clustering}

FCM clustering algorithm is different from the other two clustering algorithm used in this study. As name suggests, the algorithm utilizes the concept of fuzzy. Unlike the other two algorithms FCM does not assign a data to a particular group. Instead, FCM assign each data point with a membership function, which value ranges between 0 and 1 . This membership function depicts belongingness of a particular data point with all the groups.

A $n \times c$ matrix $U=\left[\mu_{i k}\right]$ represents the fuzzy partitions, its conditions are given by:

$$
\begin{aligned}
& \mu_{i k} \in[0,1], 1 \leq i \leq n, 1 \leq k \leq c \\
& \sum_{k=1}^{c} \mu_{i k}=1,1 \leq i \leq n \\
& 0<\sum_{i=1}^{N} \mu_{i k}<n .
\end{aligned}
$$

The FCM clustering algorithm is based on the minimization of an objective function called $c$-means functional. It is defined by Dunn (1974) as:

$$
J(X, U, V)=\sum_{i=1}^{c} \sum_{k=1}^{n}\left(\mu_{i k}\right)^{m}\left\|X_{k}-V_{i}\right\|_{A}^{2}
$$

where: $V=\left[V_{1}, V_{2}, V_{3}, \ldots, V_{c}\right], V_{i} \in R^{n}$ is a vector of cluster centres; $X$ is the data set; $U$ is the partition matrix; $V_{i}$ is the mean of data points over cluster $i ; m$ is the weight exponent which determines the fuzziness of the clusters (default value is 2 ); $n$ is the number of observations; $c$ is the number of clusters.

\subsubsection{Genetic Algorithm}

The quality of cluster result is determined by the sum of distances from objects to the centres of clus- ters with the corresponding membership values: $J=\sum_{i=1}^{c} \sum_{k=1}^{n}\left(\mu_{i k}\right)^{m} d\left(V_{i}, X_{k}\right)$, where $d\left(V_{i}, X_{k}\right)$ is the Euclidean distance between the objects.

The local minimum obtained with the fuzzy $c$ means algorithm often differs from the global minimum. Due to large volume of calculation realizing the search of global minimum of function $J$ is difficult. GA which uses the survival of fittest gives good results for optimization problem. GA does not guarantee if the global solution will be ever found, but they are efficient in finding a 'sufficiently good' solution within a 'sufficient short' time.

\subsection{SOM in ANN Clustering}

Yang and Qiao (1998) applied SOM of ANN for the pattern recognition of traffic flow states into some distinctive clusters. The accuracy of prediction of traffic volume of future traffic improved significantly with this combined algorithm. In SOM, a set of nodes is arranged in a geometric pattern which is typically a 2-dimensional lattice. Each node is associated with a weight vector with the same dimension as the input space. The purpose of the SOM is to find a good mapping. During training, each node is presented to the map so as the input data associated with it. An input weight vector of same dimension as that of input data dimension was given. The clustering using SOM algorithm was done in two steps.

Step 1. The input data is compared with all the input weight vectors $m_{i}(t)$ and the Best Matching Unit (BMU) on the map is identified. The $B M U$ is the node having the lowest Euclidean distance with respect to the input pattern $x(t)$. The final topological organization of the map is heavily influenced by this distance. $B M U m_{c}(t)$ is identified by:

$$
\text { For all } i,\left\|x(t)-m_{c}(t)\right\| \leq \| x(t)-m_{i}(t) \text {. }
$$

Step 2. Weight vectors of BMU are updated as:

$$
m_{i}(t+1)=m_{i}(t)+\alpha h_{b(x) i}\left(x(t)-m_{i}(t)\right),
$$

where: $h_{b(x)}$ is the neighbourhood function, which is:

$$
h_{b(x)}=\alpha(t) e^{\left(-\frac{\left\|r_{i}-r_{b(x)}\right\|^{2}}{2 \sigma^{2}(t)}\right)},
$$

where: $0<\alpha(t)<1$ is the learning rate factor which decreases with each iteration; $r_{i}, r_{b(x)}$ are the locations of neuron in the input lattice; $\sigma(t)$ defines the width of the neighbourhood function.

The above two steps were repeated iteratively till the pattern in input was processed.

\subsection{Validation Measure}

Cluster validity is concerned with checking the quality of clustering results. It has been mainly used to evaluate and compare whole partitions, resulting from different algorithms or resulting from the same algorithms under different parameters. Common application of cluster validation measure is to determine the correct number 
of cluster for a set of data. Different validity measures have been proposed in the literature, none of them is perfect by oneself, and therefore several indices are used in this study, such as: Silhouette Index (Rousseeuw 1987), Davies-Bouldin Index (Davies, Bouldin 1979), Caliński-Harabasz Index (Caliński, Harabasz 1974) and Dunn Index (Dunn 1973).

\subsubsection{Silhouette Index (SI)}

This index was proposed by Rousseeuw (1987) to evaluate clustering results. Silhouette width is a composite index which reflects the compactness and separation of the clusters. For each data point $i$ the Silhouette width is calculated as follows:

$$
S(i)=\frac{b(i)-a(i)}{\max \{a(i), b(i)\}},
$$

where: $a(i)$ is the average distance of a data point $i$ to other data point in the same cluster; $b(i)$ is the average distance of the that particular data point to all the data points belonging to the nearest cluster.

The average $S(i)$ of all data points reflects the quality of clustering result. Larger silhouette value signifies good cluster.

\subsubsection{Davies-Bouldin Index (DBI)}

This index is a function of the ratio of the sum of withincluster scatter to between-cluster separation. This DB index is defined by (Davies, Bouldin 1979):

$$
D B I=\frac{1}{c} \sum_{i, j=1}^{c} \max _{i \neq j}\left(\frac{D_{c}(i)+D_{c}(j)}{d_{c e}(i, j)}\right),
$$

where: $D_{c}$ and $d_{c e}$ are intra-cluster and inter-cluster distances respectively,

$$
\begin{aligned}
& D_{c}=\frac{\sum_{i}\left\|X_{a}-C_{i}\right\|}{N_{i}} ; \\
& d_{c e}=\left\|C_{i}-C_{j}\right\| .
\end{aligned}
$$

The intra-cluster distances are calculated by average of pair wise distances from points in the cluster to the cluster centroid. The inter-cluster distance between two clusters is computed as the distance between their centroids. $X_{a}$ any chosen arbitrary data that belongs to cluster i. $N_{i}$ is number of data that belong to cluster $i$. $C_{i}$ and $C_{j}$ cluster centre of $i$ and $j$ cluster. So, when the cluster is compact and far from each other the ratio is small. Consequently, Davies-Bouldin index is small for good cluster.

\subsubsection{Caliński-Harabasz Index (CHI)}

Caliński and Harabasz (1974) suggested the index for cluster validation purpose. This index uses the quotient between the intra-cluster average squared distance and inter-cluster average squared distance:

$$
F=\frac{\left(\sum_{i=1}^{n}\left(X_{i}-\bar{X}\right)^{2}-\sum_{k}^{c} \sum_{i=1}^{n_{k}}\left(X_{i}-\bar{X}_{k}\right)^{2}\right) /(c-1)}{\left(\sum_{k}^{c} \sum_{i=1}^{n_{k}}\left(X_{i}-\bar{X}_{k}\right)^{2}\right) /(n-c)},
$$

where: $n$ is total number of input data points; $c$ is number of cluster. $n_{k}$ is number of data points in cluster $k$ $(k=1,2, \ldots, c) ; X_{i}$ and $\bar{X}_{k}$ are observation vectors for input data $i$ and the centroid for group $k$, respectively. This parameter was found to be the best global statistic criterion in cluster evaluation by Milligan and Cooper (1985).

For Caliński-Harabasz Index most separated cluster value indicates the optimum number of cluster.

\subsubsection{Dunn Index (DI)}

The index was formulated by Dunn (1973) in order to check the quality of cluster resulted from a clustering algorithm. The equation is defined by:

$$
D_{n c}=\min _{i=1, \ldots, n c}\left(\min _{j=i+1, \ldots, n c}\left(\frac{d\left(c_{i}, c_{j}\right)}{\max _{k=1, \ldots, n c}\left(\operatorname{diam}\left(c_{k}\right)\right)}\right)\right),
$$

where: $d\left(c_{i}, c_{j}\right)$ is the dissimilarity function between two clusters $c_{i}$ and $c_{j}$ defined as $d\left(c_{i}, c_{j}\right)=\min _{x \in C_{i}, y \in C_{j}} d(x, y)$; $\operatorname{diam}(c)$ is the diameter of a cluster which is the measure of dispersion of the clusters. The $\operatorname{diam}(c)$ of the cluster can be defined as follows $\operatorname{diam}(c)=\max _{x, y \in C} d(x, y)$.

The larger Dunn index value indicates the presence of compact and well separated clustering.

\section{Methodology}

Off-street pedestrian facility serves only non-motorized traffic and is separated from motor vehicle traffic to the extent that such traffic does not affect their quality of service. There are different categories of exclusive pedestrian facilities: walkways, cross-flow areas, queuing area, underpass, overpass, stairways etc. The LOS thresholds for each category are different, but all are based on the concept of space per pedestrian, which is a measure of pedestrian comfort and mobility. The HCM (Highway Capacity Manual 2010) methodology for determining PLOS categories is followed in this study. The steps taken to determine the LOS of exclusive off-street walkways pedestrian facilities are as follows.

\section{Step 1. Determination of Effective Walkway Width}

Effective walkway width is the portion of a walkway that can be used effectively by pedestrians. Various types of obstructions and linear features, discussed below, reduce the walkway area that can be effectively used by pedestrians. The effective walkway width at a given point along the walkway is computed as follows:

$$
W_{E}=W_{T}-W_{O} \text {, }
$$

where: $W_{E}$ - effective walkway width; $W_{T}$ - total walkway width at a given point along walkway; $W_{O}$ - sum of 
fixed-object effective widths and linear feature shy distances at a given point along walkway.

\section{Step 2. Calculation of Pedestrian Flow Rate}

An hourly pedestrian demand is used as an input to the analysis. Consistent with the general analysis procedures used throughout the HCM, hourly demand is usually converted into peak 15 min flows, so that LOS is based on the busiest 15 consecutive minutes during an hour:

$$
V_{15}=\frac{V_{h}}{4 \cdot P H F},
$$

where: $V_{15}$ - pedestrian flow rate during peak $15 \mathrm{~min}$ [ped/h]; $V_{h}$ - pedestrian demand during analysis hour [ped/h]; PHF - peak hour factor.

However, if peak 15 min pedestrian volumes are available, the highest $15 \mathrm{~min}$ volume can be used directly without the application of a peak hour factor. Next, the peak 15 min flow is converted into a unit flow rate per effective path width $[\mathrm{ped} / \mathrm{sec} / \mathrm{m}]$ :

$$
V_{P}=\frac{V_{15}}{15 \cdot W_{E}},
$$

where: $V_{P}$ - pedestrian flow per unit width [ped $/ \mathrm{m} / \mathrm{sec}$ ] and all other variables are as previously defined.

\section{Step 3.1. Calculation of Average Pedestrian Space}

The service measure for walkways is pedestrian space, the inverse of density. Pedestrian space can be directly observed in the field by measuring a sample area of the facility and determining the maximum number of pedestrians at a given time in that area. The pedestrian unit flow rate is related to pedestrian space and speed:

$$
A_{P}=\frac{S_{P}}{V_{P}},
$$

also Density $=1 / A_{P}$,

where: $A_{P}$ - pedestrian space $\left[\mathrm{m}^{2} /\right.$ ped]; $S_{P}$ - pedestrian speed $[\mathrm{m} / \mathrm{sec}] ; V_{P}$ - pedestrian flow per unit width $[\mathrm{ped} / \mathrm{m} / \mathrm{sec}]$.

\section{Step 3.2. Volume to Capacity ratio (v/c) Calculation}

For determination of PLOS category of off-street pedestrian facility volume to capacity $(v / c)$ ratio is one of the most important factor. For this study pedestrian hourly volume can be found out from video data collection and capacity of side-walks has been taken from Indian Road Congress (IRC: 103-1989). Here width of side-walk for $1.5 \mathrm{~m}, 2 \mathrm{~m}, 2.5 \mathrm{~m}, 3 \mathrm{~m}$ and $4 \mathrm{~m}$ capacities in number of persons per hour in both directions are $800,1600,2400,3200$ and 4000 respectively.

\section{Step 4. Determine Pedestrian Level of Service (PLOS)}

PLOS categories are to be resolute on the basis of average pedestrian space $\left(A_{p}\right)$. Six no of PLOS designated as 'A' to ' $\mathrm{F}$ ' has to determine for off-street pedestrian facilities. Not only on the basis of average space, other related measures like flow rate, average space and volume by capacity ratio are also considered for categorisation of PLOS.

\section{Study Corridors and Data Collection}

A century of industrialization and technical advancement has brought forth rapid urbanization in India. Statistics on census of India 2011 reveals that about 377 million of the total 1.21 billion population of India lives in urban areas. As per the census Odisha is having 41 million populations, that is $3.14 \%$ of population of India and the population is closest to country Argentina. However, decadal growth in the urban population of the State, during the last decade (2001-2011) has been enormous with a growth rate of about $26.80 \%$, which is nearly that of national growth rate of $31.80 \%$. It is noteworthy that the state's population during the last decade has grown by about $13.97 \%$ while that of the urban population has grown at almost at double this rate.

Two important mid-size cities Bhubaneswar and Rourkela of Odisha state, India, are taken up in the present study. Bhubaneswar, the temple city enjoys excellent connectivity with other adjoining regions of strategic importance - however, the passenger transit option needs improvement of greater interaction. Bhubaneswar is primarily an administrative city and a tourism city. Bhubaneswar has emerged as a fast-growing, important trading and commercial hub in the state and eastern India. Tourism is a major industry, attracting about 1.5 million tourists in 2011. Bhubaneswar was designed to be a largely residential city with outlying industrial areas. The economy had few major players until the 1990s and was dominated by retail and small-scale manufacturing. There exists a significant level of disparity within this region in terms of accessibility to major urban centres. At intra urban level, incapacity of the existing traffic and transportation network will create a serious constraint to its future growth. The traffic demand management will play a key role as the role of supply management is near exhaustion. On the other hand, Bhubaneswar enjoys a variable level of mobility at different parts of the city. Rourkela, the site for this study is commonly known as the steel city in all over world is one of the largest cities located at northern west of the Odisha state. It is situated at the heart of mineral belt. The correct selection of sample sites is an important issue in designing the field survey. In reality, selection of a proper site is not an easy task because different routes need to appear in different LOS conditions to examine the influence of LOS on pedestrian route choice. Sampled area sees heavy foot traffic, because of sidewalks on the streets and transit points. Riders of public transportation (subway, bus, train) usually walk from transit points to their destinations. Another feature of the selected area is the great variety of land uses: residential, commercial, office and institutional (e.g. school, hospital). Often, commuters need to walk at least a short distance to reach their final destinations. Bhubaneswar and Rourkela has straight, wide streets, unlike the narrow, winding, ancient streets found in most Indian cities. Almost all the streets have sidewalks that are separated from the carriageway by small trees and curbs. Since bicycle lanes have not been established in cities, cyclists also use these 
sidewalks. Streets are arranged in a grid, meeting at signalized intersections that are installed with pedestrian crosswalks. In the surveyed area, almost all the streets have sidewalks on both sides and crosswalks at intersections. Study corridors for the study area are presented in Fig. 1. The locations of the two cities (Bhubaneswar and Rourkela) are shown in Fig. 1a. The road corridors of Bhubaneswar and Rourkela cities are given by Fig. $1 \mathrm{~b}$ and Fig. 1c respectively.

Increasingly, researchers are using video camera to observe and collect data about pedestrians. Video data has plenty of advantages over direct observation: one can collect data from the video carefully back in the office or lab, can easily share video with others to illustrate a point, and there are tools available to automate data collection. High resolution video camera was fixed to the side of the foot-path that is the pedestrian off-street facilities with the help of a tripod stand. Always a length of $4 \mathrm{~m}$ has been marked to observe the flow of pedestrian. However the start and end point of the stretch having four meter length of the footpath helped in determining the speed of the pedestrian.

Pedestrian speed and attributes on road features data were collected on a sample of 3764 pedestrians observed at various sidewalk locations in both of the cities about 62 days in both peak and off-peak hours of working and non-working days. In Bhubaneswar city from the locations like Rajpath, Mahatma Gandhi Road, Master Canteen area, Sachivalay Marg (AG square to PMG square), KIIT road, Magnetic square, OUAT square, BJB college square, Vanivihar to Rupali square, Acharya vihar and Khandagiri square, video data samples were collected. Considering about Rourkela city different locations like Bisra chowk to Sail square, Main Market, Station square, Daily market, STI Chowk, Panposh road and Ambagan market etc., off-street pedestrian video data were collected. Some photos of walkways of the study corridor are shown in Fig. 2.

This shows that a large size data set is used for the development of LOS categories in this study. The speed of each pedestrian is recorded by stopwatch. With the help of stopwatch pedestrian crossing time from the starting point to the end point of the observed portion of the off-street segment was recorded. Noting down the crossing time of each walker, the pedestrian speed per meter is extracted. For the further calculation average pedestrian speed for each segment were intended. It is assumed that the average speed of pedestrians on the observed section of the facility represents the average speed of the street segment as a whole. In the same locations, over the same time period, all pedestrians were counted in order to determine sidewalk flow rates, and basic information about each of the 31 station points was recorded.

Then the videos were loaded to computer to play the recorded videos. The pedestrian peak volume and flow rate from video data were calculated manually. It is observed that in these two cities pedestrian flow and density are comparatively lower because population sizes of these two cities are less than a million and also mobility due to commercial activities is comparatively lower.

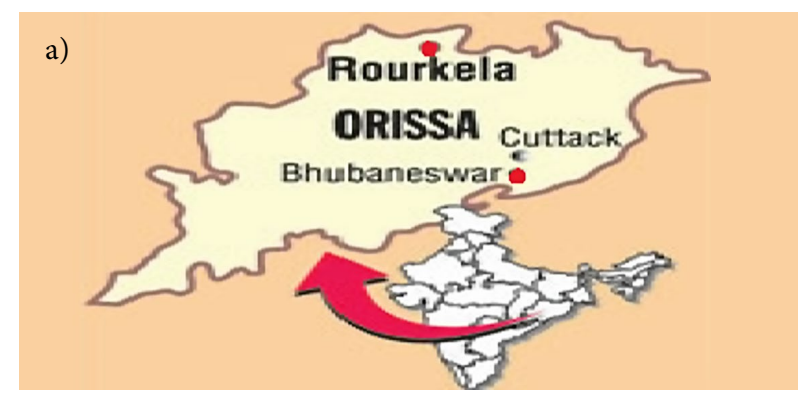

b)

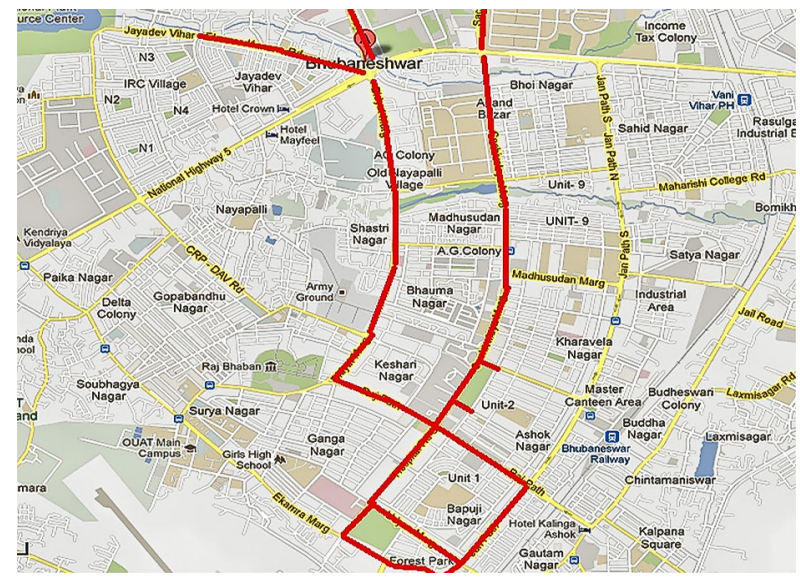

c)

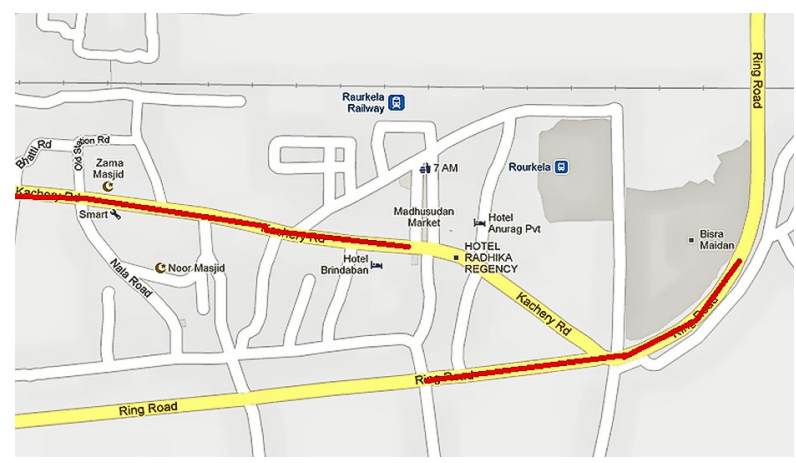

Fig. 1. Map showing location of the cities and the road corridors of the study areas: $\mathrm{a}$ - location of the cities; $\mathrm{b}$ - Bhubaneswar study corridors; c - Rourkela study corridors

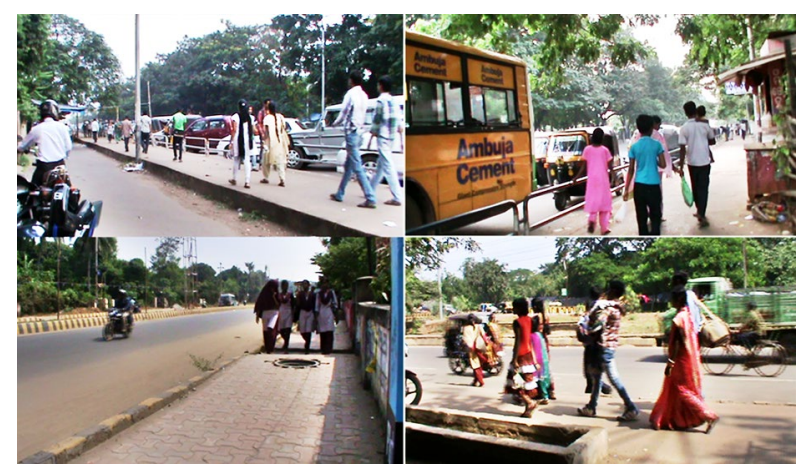

Fig. 2. Schematic photographs of walkways in the study locations 
Based on these sets of data, two databases were built: a database containing speed and an aggregate database of each of the study locations. This aggregated locational database includes the calculated flow rate based on the count at the location, the effective width of the sidewalk, and land use proportions based on two cities. Effective walkway width is the portion of a walkway that can be used effectively by pedestrians. Various types of obstructions such as trees, electric poles, information sign boards, projections of road side shops and linear features, reduce the walkway area that could have effectively used by pedestrians.

\section{Results and Analysis}

\subsection{Validation Measure for Optimal Number of Cluster}

Four cluster validation parameters (SI, DBI, CHI, DI) were analyzed in this research to determine the most suitable clustering algorithm for classification of average space data. For determination of the parametric values of validation measures, four measuring parameters (average pedestrian space, flow rate, speed and $v / c$ ratio) data acquired through Video Camera was clustered using AP, GA-Fuzzy and SOM cluster analysis is used as input to the validation parameters. In this study data set of average pedestrian space is explained in detail and all other parameters are also observed in the same manner. By knowing the optimum number of clusters we can classify the urban off-street pedestrian facilities into that number of LOS categories. It is always considered that lesser number of clusters is better if variation in validation parameters is minimal. Values of validation parameters are obtained for 2 to 7 number of cluster are plotted in Fig. 3.

Literature shows that the largest silhouette value indicates a better quality of clustering result. Fig. 3a shows that the index value for all three cluster analysis are largest for 6 number of cluster. Also available literature says that for DBI a low value indicates the optimum number of cluster. From the Fig. $3 \mathrm{~b}$ it is observed that index value is minimum for 6 number of cluster. Literature shows the maximum separation in the cluster values shows the optimum number of cluster. Fig. $3 \mathrm{c}$ shows that the optimum number of cluster for $\mathrm{CHI}$ is 6 . From some literature it is found that the maximum value of DI represents the optimum number of cluster. But in case of DI we can see in Fig. $3 \mathrm{~d}$ the optimum number of cluster is different for different cluster analysis.

Using average pedestrian space as the Measure Of Effectiveness (MOE), three validation indices (SI, DBI, $\mathrm{CHI}$ ) justify the optimal clusters as 6; which is also same as suggested by HCM (Highway Capacity Manual 2010). Other three MOEs (pedestrian flow rate, speed and volume to capacity ratio) also satisfy classification of PLOS into six categories. That is the reason for which in this research the urban off-street pedestrian facilities are classified into six categories using AP, GA-Fuzzy and SOM cluster analysis methods. The different ranges of the parameters are given in different symbols; also the legends in figures give the ranges.
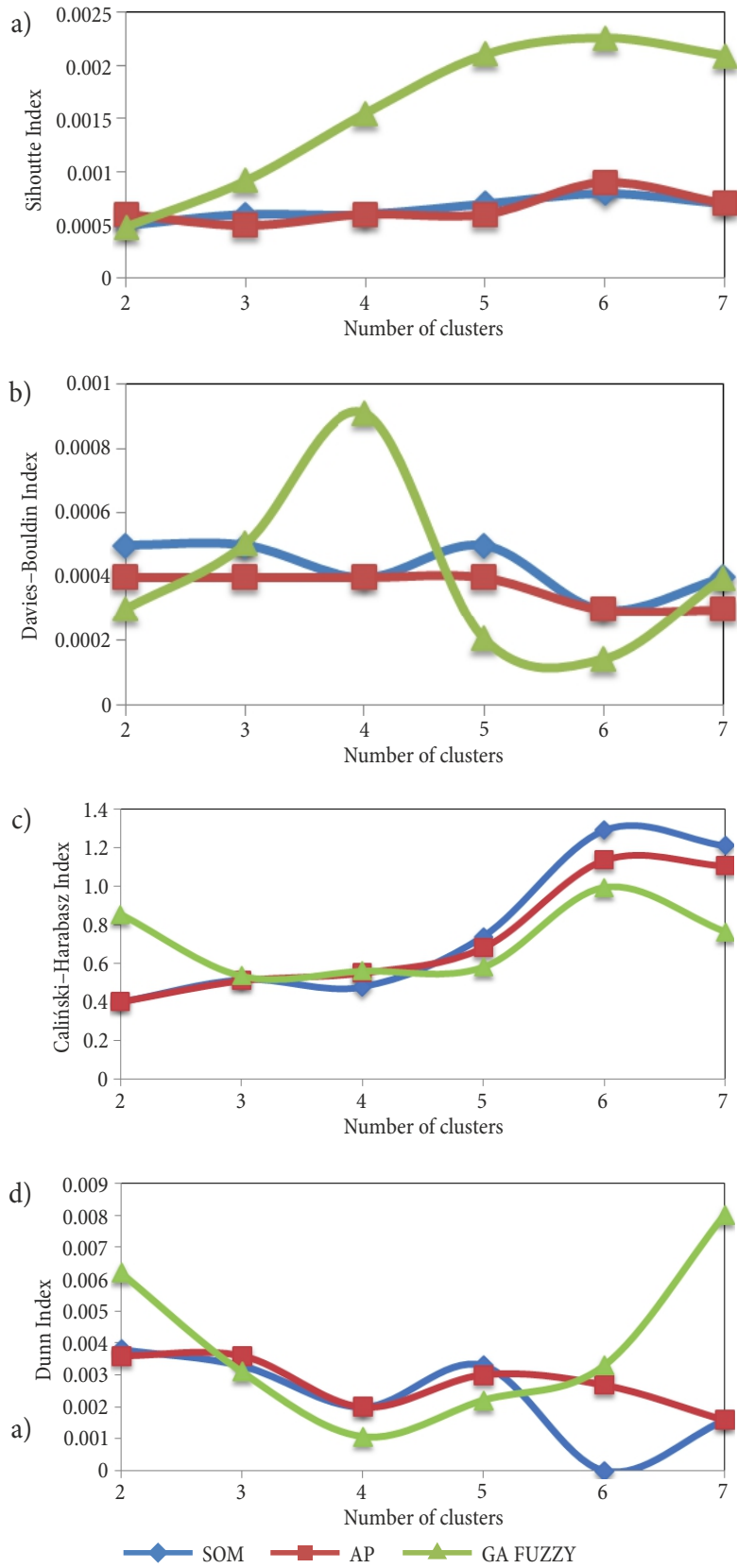

Fig. 3. Values of validation parameters for different number of clusters using cluster algorithms: a - Silhouette Index vs number of cluster; $\mathrm{b}$ - Davies-Bouldin Index vs number of cluster; c - Caliński-Harabasz Index vs number of cluster; $\mathrm{d}$ - Dunn Index vs number of cluster

\subsection{Selection of Appropriate Cluster Method}

To compare the results of each clustering methodology Oh and Ritchie (2002) used the concept of Wilk' Lambda. Wilk's Lambda $(\lambda)$ is defined as the ratio of within group's variance to total variance. A lower Wilk's lambda (W-lambda) represents better clustering:

$$
\lambda=\frac{|W|}{|T|}=\frac{|W|}{|B+W|},
$$

where: $W$ - pooled within-groups variance; $B$ - between groups variance; $T$ - total variance. 
Average pedestrian space, flow rate, pedestrian speed and volume to capacity ratio were used for clustering. W-lambda for clustering values of all the four parameters is observed. Fig. 4. shows the clustering approach of average pedestrian space.

As we can identify in the clustering results, the overall values of Wilk's Lambda from GA-Fuzzy clustering are lower than the other methods, so the threshold values were determined be GA-Fuzzy clustering for average pedestrian space. It is also observed that for other three measuring parameters $\mathrm{W}$-lambda is having lower values for the GA-Fuzzy clustering than the other two clustering method. Based on these results, PLOS ranges of the four parameters are defined using GA-Fuzzy clustering.
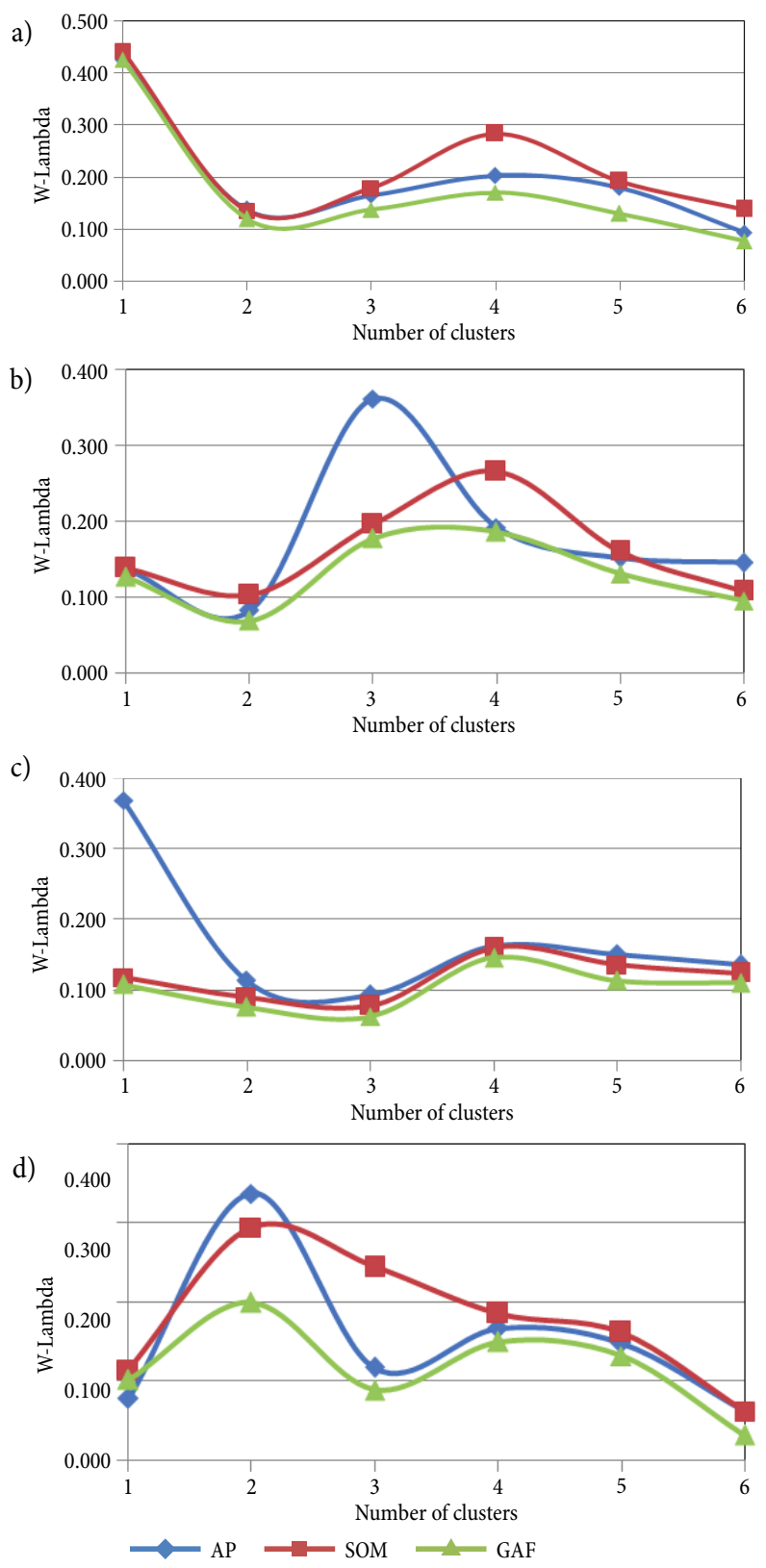

Fig. 4. Values of W-lambda for different number of cluster algorithms: $\mathrm{a}-\mathrm{W}$-lambda for pedestrian space; $\mathrm{b}-\mathrm{W}$-lambda for flow rate; $\mathrm{c}-\mathrm{W}$-lambda for speed; $\mathrm{d}-\mathrm{W}$-lambda for $v / c$

\subsection{Cluster Analysis}

\section{GA-Fuzzy Clustering}

The most popular method in fuzzy classification called GA-Fuzzy is used for defining PLOS criteria of off-street pedestrian facilities. GA is used for optimization of search, to get the local minima which differ from global minima. The search for the global minimum cannot be realized due to a large volume of calculations, but GA is used to get a sufficiently good solution. For determination of the PLOS criteria for off-street pedestrian facility, pedestrian space, speed, $\mathrm{f}$ low rate and $v / c$ ratio data acquired through video data are used. Fig. 5 and Table 2 describe the PLOS ranges of off-street pedestrian facilities found from GA-Fuzzy clustering.

From GA-Fuzzy cluster analysis it is found that for PLOS 'A' average pedestrian space is greater than $16.53 \mathrm{~m}^{2} / \mathrm{ped}$ and for PLOS ' $\mathrm{F}$ ' it is less than $4.48 \mathrm{~m}^{2} /$ ped. Pedestrians will face frequent contact with other users and speed is restricted when flow rate is greater than $0.1146 \mathrm{ped} / \mathrm{sec} / \mathrm{m}$ and at that time volume to capacity ratio is at extreme level i.e. near about 1 . It is also observed that pedestrians can move at their desired speed at less than $0.061 \mathrm{ped} / \mathrm{sec} / \mathrm{m}$ flow rate and less than $0.4 \mathrm{v} / \mathrm{c}$. This occurs in PLOS 'A' condition which is the free flow state and here pedestrians move at near about $1.21 \mathrm{~m} / \mathrm{sec}$ speed. LOS A represents the best operating conditions from the traveller's perspective and LOS ' $F$ ' the worst. For cost, environmental impact, and other reasons, roadways are not typically designed to provide LOS 'A' conditions during peak periods, but rather some lower LOS that reflects a balance between individual travellers' desires and society's desires and financial resources.

a)

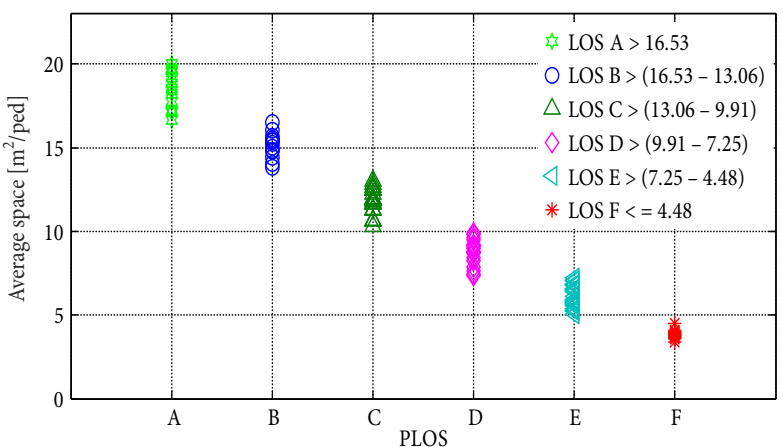

b)

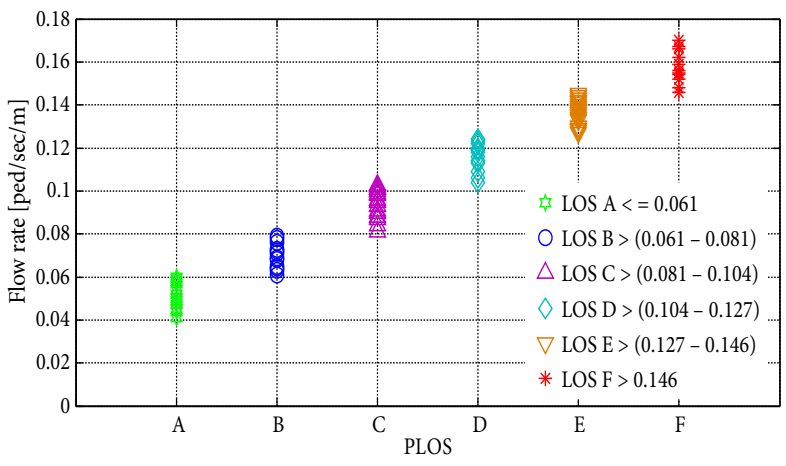

To be continued 
c)

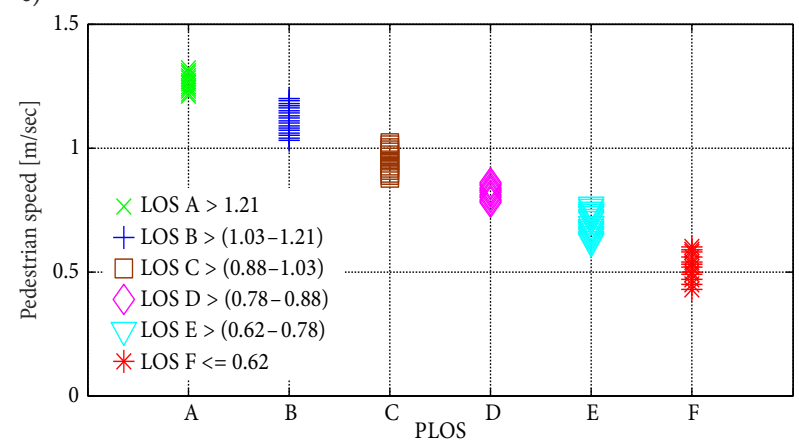

d)

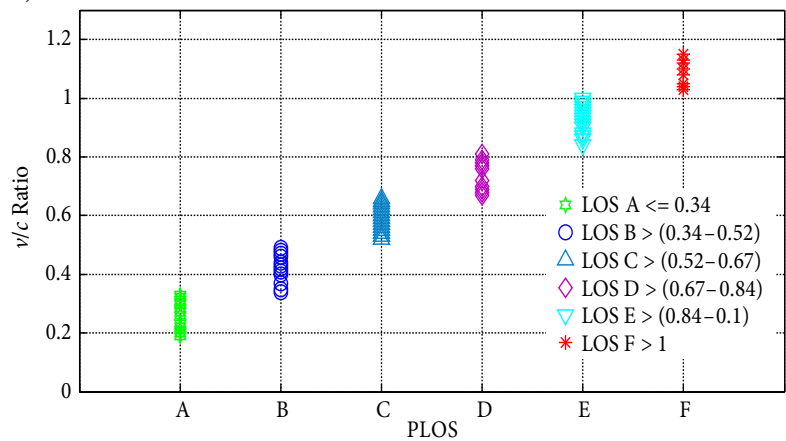

Fig. 5. PLOS Categories of urban off-street pedestrian facilities using AP clustering on various parameters: $a$ - average pedestrian space of PLOS categories; $b$ - flow rate of PLOS Categories; $c-$ average travel speed of PLOS categories; $d-v / c$ ratio of PLOS categories

Table 2. PLOS Categories of urban off-street pedestrian facilities using AP clustering on various parameters

\begin{tabular}{|c|c|c|c|c|l|}
\hline \multirow{2}{*}{ LOS } & $\begin{array}{c}\text { Average space } \\
{\left[\mathrm{m}^{2} / \mathrm{ped}\right]}\end{array}$ & \multicolumn{3}{|c|}{ Related measures } & \multicolumn{2}{|c|}{ Comments } \\
\cline { 3 - 5 } & $>16.53$ & $\leq 0.061$ & $>1.21$ & $\leq 0.4$ & $\begin{array}{l}\text { Ability to move in desired path, no need } \\
\text { to alter movements }\end{array}$ \\
\hline 'A' & $>13.06 \div 16.53$ & $>0.061 \div 0.081$ & $>1.03 \div 1.21$ & $>0.4 \div 0.57$ & $\begin{array}{l}\text { Occasional need to adjust path to avoid } \\
\text { conflicts }\end{array}$ \\
\hline 'C' & $>9.91 \div 13.06$ & $>0.081 \div 0.104$ & $>0.88 \div 1.03$ & $>0.57 \div 0.76$ & $\begin{array}{l}\text { Frequent need to adjust path to avoid } \\
\text { conflicts }\end{array}$ \\
\hline 'D' & $>7.25 \div 9.91$ & $>0.104 \div 0.127$ & $>0.78 \div 0.88$ & $>0.76 \div 0.9$ & $\begin{array}{l}\text { Speed and ability to pass slower } \\
\text { pedestrians restricted }\end{array}$ \\
\hline 'E' & $>4.48 \div 7.25$ & $>0.127 \div 0.146$ & $>0.62 \div 0.78$ & $>0.9 \div 1.00$ & $\begin{array}{l}\text { Speed restricted, very limited ability to } \\
\text { pass slower pedestrians }\end{array}$ \\
\hline 'F' & $\leq 4.48$ & $>0.146$ & $\leq 0.62$ & $>1$ & $\begin{array}{l}\text { Speed severely restricted, frequent contact } \\
\text { with other users }\end{array}$ \\
\hline
\end{tabular}

Nevertheless, during low-volume periods of the day, a system element may operate at LOS 'A'. PLOS 'B' also perform a good facilities for the pedestrians flow where walkers are having $13.06 \mathrm{~m}^{2} /$ ped average space and they can move with a speed of $1.03 \mathrm{~m} / \mathrm{sec}$. In LOS 'B' very rarely road users have to change or alter their path. As discussed in the data collection part, these two cities are having low population densities for which wide ranges of measuring parameters are observed for six LOS categories. From the speed data evaluation it is observed that speed of pedestrian ranges from 0.45 to $1.32 \mathrm{~m} / \mathrm{sec}$ which practically occurs due to the variation of places and pedestrian behaviour. In the market areas pedestrian moves in a high speed but sometimes some people move in a casual manner, with a very slow speed.

\subsection{Fundamental Diagram}

From the video recording survey, the observed capacities for the off-street pedestrian facilities were obtained. The pedestrian speed, flow rate and pedestrian density at the facilities are also determined. As walkways are exposed to worse environments (e.g. high temperature, wind, rain, dust, etc.) and these would cause the pedestrians to walk faster. Due to the nature of the pedestrian movements on these facilities on-concourses, pedestrian movements are complex and multimodal movements are observed, whereas on some places pedestrian movements are pre- dominantly bidirectional, hence higher maximum flow rates are observed. To know about the kind of relationship that exists among the fundamental parameters (Speed, flow and density) for off-street facilities curves are plotted in the Fig. 6. From Fig. 6a, it has been observed that a linear relationship of reverse order exist between pedestrian density $\left[\mathrm{ped} / \mathrm{m}^{2}\right]$ and pedestrian speed $[\mathrm{m} / \mathrm{sec}]$. Fig. $6 \mathrm{~b}$ is about the relation between pedestrian flow rate and speed and Fig. $6 \mathrm{c}$ is the relation between flow rate and density.

The variation in walking speed is large when the pedestrian flow is low. Under such circumstances, pedestrians can easily control their walking speeds, and therefore they can overtake or to be easily overtaken by others. On the other hand, the variations in walking speed are small if the pedestrian flow is high. As pedestrian can no longer control their own walking speed, their locomotion will be largely affected by pedestrians around them. Maximum and minimum densities are observed in different places and the attributes causing such behavior might be the level of friction experienced on the road and the roadway width. The higher level of friction and lesser roadway width make the pedestri- 
a)

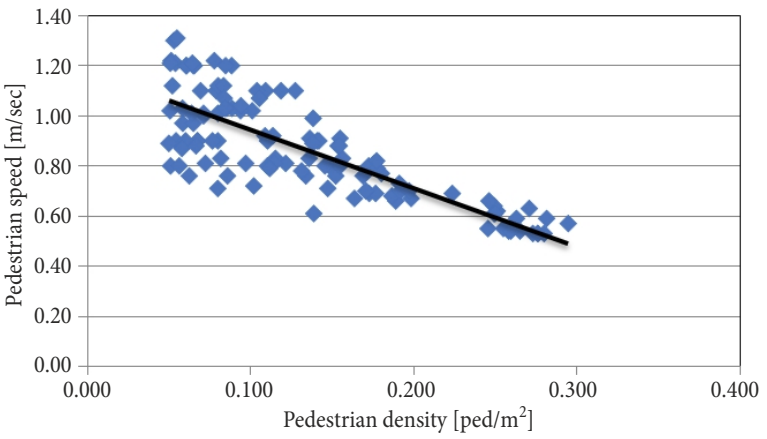

b)

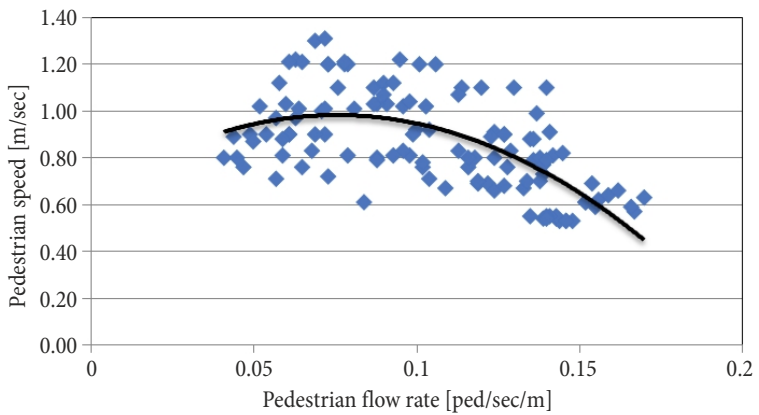

c)

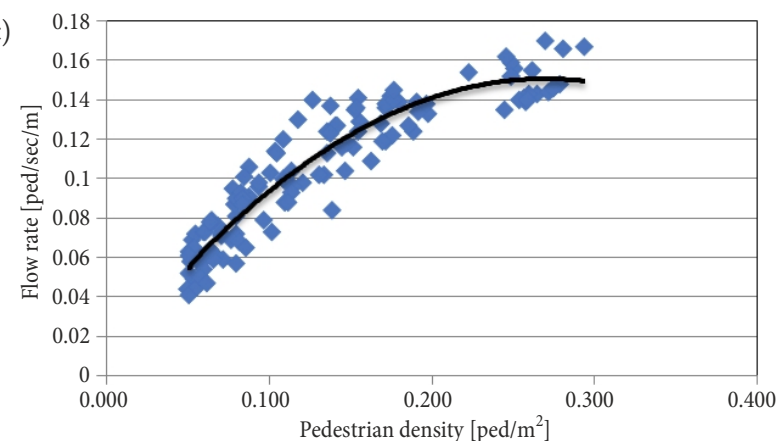

Fig. 6. Pedestrian flow characteristics for off-street pedestrian facilities: a - density vs speed diagram; b - flow rate vs speed diagram; c - flow rate vs density diagram

ans to use restricted road space, thus resulting in higher density. The variation in maximum pedestrian flow rates followed the trend shown by maximum pedestrian density. The maximum and minimum flow rates are observed as $0.146 \mathrm{ped} / \mathrm{sec} / \mathrm{m}$ and 0.061 respectively. It is observed that the pedestrian density increases linearly as the pedestrian flow rate increase and after reaching a peak level of flow the flow rate decreases as the density increases further. From Table 2 it can be observed that walker get poor kind of service ' $F$ ' when flow rate is $0.146 \mathrm{ped} / \mathrm{sec} / \mathrm{m}$ and above and walker enjoys good service quality 'A' when flow rate is $0.061 \mathrm{ped} / \mathrm{sec} / \mathrm{m}$ and below of it.

\section{Conclusions}

In this study an attempt has been made to define PLOS categories of urban off-street pedestrian facilities for developing countries like India. Recently developed analysis techniques such as AP, SOM in ANN and GA-Fuzzy clustering are used in this regard. From the available source it is found that HCM (2010) methodology for the prediction of PLOS can be used for Indian context after due modifications. Hence, the PLOS methodology developed in HCM (Highway Capacity Manual 2010) is adopted in this study. Different LOS values based on pedestrian space, flow rate, speed of pedestrian and volume to capacity $(v / c)$ ratio are defined from each clustering analysis method which gives numeric ranges for LOS categories. An in-depth research is carried out by applying W-lambda on four measuring parameters to choose the most suitable clustering method in defining PLOS criteria that is well applicable in Indian context. While comparing these clustering methods it is found that GAFuzzy clustering has the minimum value of W-lambda for all the four parameters used in defining PLOS criteria. Hence, it is well convinced that GA-Fuzzy is the most suitable clustering method that can be applied for this purpose. From this study it is observed that pedestrian data collection using video cameras is a very simple and accurate procedure. Also, GA-Fuzzy clustering is highly efficient in terms of time saving and provides a very accurate solution to this kind of classification problem. By using cluster analysis, ranges of parameters for six PLOS categories i.e. 'A', 'B', 'C', 'D', ' $\mathrm{E}$ ' and ' $\mathrm{F}$ ' are defined for off-street walking facility in Indian context; where LOS 'A' represents the best operating conditions and LOS ' $F$ ' the worst. The PLOS ranges defined in this study are significantly different from that mentioned in HCM (Highway Capacity Manual 2010).

In this study, these two cities are having less than a million population, for which pedestrian movement is comparatively low than the highly populated metropolitan cities. In Indian cities highly heterogeneous traffic flow on the main carriageway occasionally influence the pedestrian's movement on off-street facilities. Due to poor enforcement of laws for traffic on main carriageways as well as the off-street pedestrian facilities, a haphazard movement is perceived. Also it has been observed that inadequate road infrastructures lead to varying geometry conditions that create unwanted confusion to the users. Besides, some of the pedestrian facilities are unauthorized and occupied by vendors for their commercial use and installation of advertisements boards. In some cases unplanned utilities, such as electric and telephone poles become a natural obstruction to the pedestrian movements on the path. Illegal parking on off-street facilities becomes a common phenomenon for which the pedestrian has to forcefully reduce its speed and divert the direction of movement. For these reasons PLOS ranges in Indian cities are different from other developed countries. In India, social and cultural inheritance is also different as people love to move in platoons, which has a broad effect on off-street movements. Also the physical size of Indian population is also another contributing factor for which the PLOS categories in this study are different from the values described in HCM.

The pedestrian flow characteristics are analysed at off-street pedestrian facilities in a mid-sized cities in India. The free-flow speeds are found quite high, $1.32 \mathrm{~m} / \mathrm{sec}$. The observed free-flow speed and maximum 
density are found proportional to each other. From the analysis it is concluded that with the increase in friction faced by a pedestrian, contrary to the normal belief, the pedestrian speed also increase. Considering limitation, it is proposed that similar studies can be carried out for bigger cities to develop comprehensive PLOS criteria.

\section{References}

Caliński, T.; Harabasz, J. 1974. A dendrite method for cluster analysis, Communications in Statistics 3(1): 1-27. http://dx.doi.org/10.1080/03610927408827101

Čokorilo, O.; De Luca, M.; Dell'Acqua, G. 2014. Aircraft safety analysis using clustering algorithms, Journal of Risk Research 3(1): 1-27.

http://dx.doi.org/10.1080/13669877.2013.879493

Davies, D. L.; Bouldin, D. W. 1979. A cluster separation measure, IEEE Transactions on Pattern Analysis and Machine Intelligence PAMI-1(2): 224-227.

http://dx.doi.org/10.1109/TPAMI.1979.4766909

Dueck, D.; Frey, B. J. 2007. Non-metric affinity propagation for unsupervised image categorization, in IEEE 11th International Conference on Computer Vision, ICCV 2007, 14-21 October 2007, Rio de Janeiro. 8 p.

http://dx.doi.org/10.1109/ICCV.2007.4408853

Dunn, J. C. 1973. A Fuzzy relative of the ISODATA process and its use in detecting compact well-separated clusters, Journal of Cybernetics 3(3): 32-57.

http://dx.doi.org/10.1080/01969727308546046

Fang, F. C.; Pecheux, K. K. 2009. Fuzzy data mining approach for quantifying signalized intersection level of services based on user perceptions, Journal of Transportation Engineering 135(6): 349-358.

http://dx.doi.org/10.1061/(ASCE)0733-947X(2009)135:6(349)

Highway Capacity Manual. 2010. Transportation Research Board. $1650 \mathrm{p}$.

Highway Capacity Manual. 2000. Transportation Research Board. $1134 \mathrm{p}$.

IRC: 103-1989. Guidelines for Pedestrian Facilities. Indian Roads Congress.

Jaskiewicz, F. 2000. Pedestrian level of service based on trip quality, in Urban Street Symposium Conference Proceedings, June 28-30, 1999, Dallas, Texas. 14 p. Available from Internet: http://onlinepubs.trb.org/onlinepubs/circulars/ec019/ Ec019_g1.pdf

Kaufman, L.; Rousseeuw, P. J. 2005. Finding Groups in Data: An Introduction to Cluster Analysis. John Wiley \& Sons Inc. http://dx.doi.org/10.1002/9780470316801

Kim, K.; Hallonquist, L.; Settachai, N.; Yamashita, E. 2006. Walking in Waikiki, Hawaii: measuring pedestrian level of service in an urban resort district, Transportation Research Record 1982: 104-112. http://dx.doi.org/10.3141/1982-15

Laxman, K. K.; Rastogi, R.; Chandra, S. 2010. Pedestrian flow characteristics in mixed traffic conditions, Journal of Urban Planning and Development 136(1): 23-33.

http://dx.doi.org/10.1061/(ASCE)0733-9488(2010)136:1(23)

Lingras, P. 2001. Statistical and genetic algorithms classification of highways, Journal of Transportation Engineering 127(3): 237-243.

http://dx.doi.org/10.1061/(ASCE)0733-947X(2001)127:3(237)

Lingras, P. 1995. Classifying highways: hierarchical grouping versus Kohonen neural networks, Journal of Transportation Engineering 121(4): 364-368.

http://dx.doi.org/10.1061/(ASCE)0733-947X(1995)121:4(364)
Miller, S.; Molino, J. A.; Kennedy, J. F.; Emo, A. K.; Do, A. 2008. Segway rider behavior: speed and clearance distance in passing sidewalk objects, Transport Research Record 2073: 125-132. http://dx.doi.org/10.3141/2073-14

Muraleetharan, T.; Hagiwara, T. 2007. Overall level of service of urban walking environment and its influence on pedestrian route choice behavior: analysis of pedestrian travel in Sapporo, Japan, Transportation Research Record 2002: 7-17. http://dx.doi.org/10.3141/2002-02

Oh, C.; Ritchie, S. G. 2002. Real-Time inductive-signaturebased level of service for signalized intersections, Transportation Research Record 1802: 97-104.

http://dx.doi.org/10.3141/1802-12

Petritsch, T. A.; Landis, B. W.; McLeod, P. S.; Huang, H. F.; Challa, S.; Skaggs, C. L.; Guttenplan, M.; Vattikuti, V. 2006. Pedestrian level-of-service model for urban arterial facilities with sidewalks, Transportation Research Record 1982: 84-89. http://dx.doi.org/10.3141/1982-12

Rastogi, R.; Thaniarasu, I.; Chandra, S. 2011. Design implications of walking speed for pedestrian facilities, Journal of Transportation Engineering 137(10): 687-696. http://dx.doi.org/10.1061/(ASCE)TE.1943-5436.0000251

Rouphail, N. M.; Hummer, J. E.; Milazzo, J. S.; Allen, D. P. 1998. Recommended Procedures Chapter 13, 'Pedestrians', of the Highway Capacity Manual. Final Report No. FHWA-RD-98-107. US Department of Transportation, Federal Highway Administration, Turner-Fairbank Highway Research Center, McLean, Virginia. 56 p. Available from Internet: https://www.fhwa.dot.gov/publications/research/ safety/pedbike/98107/98107.pdf

Rousseeuw, P. J. 1987. Silhouettes: a graphical aid to the interpretation and validation of cluster analysis, Journal of Computational and Applied Mathematics 20: 53-65. http://dx.doi.org/10.1016/0377-0427(87)90125-7

Sisiopiku, V. P.; Byrd, J.; Chittoor, A. 2007. Application of levelof-service methods for evaluation of operations at pedestrian facilities, Transportation Research Record 2002: 117-124. http://dx.doi.org/10.3141/2002-15

Van Houten, R.; Ellis, R.; Kim, J.-L. 2007. Effects of various minimum green times on percentage of pedestrians waiting for midblock "walk" signal, Transportation Research Record 2002: 78-83. http://dx.doi.org/10.3141/2002-10

Xia, D.-Y.; Wu, F.; Zhang, X.-Q.; Zhuang, Y.-T. 2008. Local and global approaches of affinity propagation clustering for large scale data, Journal of Zhejiang University SCIENCE A 9(10): 1373-1381. http://dx.doi.org/10.1631/jzus.A0720058

Yang, H.; Qiao, F. 1998. Neural network approach to classification of traffic flow states, Journal of Transportation Engineering 124(6): 521-525.

http://dx.doi.org/10.1061/(ASCE)0733-947X(1998)124:6(521)

Zhou, E.; Khotanzad, A. 2007. Fuzzy classifier design using genetic algorithms, Pattern Recognition 40(12): 3401-3414. http://dx.doi.org/10.1016/j.patcog.2007.03.028 